\title{
The impact of drying on bioactive compounds of blue honeysuckle berries (Lonicera caerulea var. edulis Turcz. ex Herder)
}

\author{
Mateja Senica $^{1 \star}$, Franci Stampar $^{1}$, Sezai Ercisli², Barbara Sladonja ${ }^{3}$, Danijela Poljuha ${ }^{3}$, \\ Maja Mikulic-Petkovsek ${ }^{1}$ \\ ${ }^{1}$ University of Ljubljana, Biotechnical Faculty, Department of Agronomy, Chair for Fruit Growing, Viticulture and \\ Vegetable Growing, Jamnikarjeva 101, SI-1000, Ljubljana, Slovenia \\ ${ }^{2}$ Ataturk University, Agricultural Faculty, Department of Horticulture, 25240 Erzurum, Turkey \\ ${ }^{3}$ Institute of Agriculture and Tourism, Karla Huguesa 8, HR-52440 Poreč, Croatia
}

\begin{abstract}
Drying fruit is one of the simplest ways to extend the shelf-life of fruit, especially berries. Both higher temperature and time of heating significantly change the contents of some primary and secondary metabolites in honeysuckle fruit. Differences in their contents arising from different heat treatments were determined with the aid of high-performance liquid chromatography (HPLC) coupled with mass spectrophotometry (MS). The content of sugars showed a small change with drying, while organic acid contents decreased with a longer drying time. Ascorbic acid was totally degraded, regardless of the time or heating temperature. Different phenolic groups responded differently to heat intensity and time of drying. Flavanols were more sensitive to higher temperature than to duration of heating and they decreased by more than $70 \%$ at $75^{\circ} \mathrm{C}$. In contrast, the content of hydroxycinnamic acids, increased with drying by more than $75 \%$, regardless of the time and temperature.
\end{abstract}

Keywords: heating, Lonicera, phenolics, time of drying

\section{Introduction}

Small fruit berries, such as blueberries, strawberries, blackberries and raspberries, are widely consumed all over the world. They are a rich source of polyphenolics, especially flavonols and anthocyanins, which have health benefits (Sablani et al. 2010, Mikulic-Petkovsek et al. 2012). Minor fruits, such as quince, rose hip, hawthorn, saskatoon, chokeberries and honeysuckles, are easier to grow and hardy in nature, producing a crop even under adverse soil and climatic conditions (Gündüz and Özbay 2018). Their fruits have a unique aroma and taste and play a vital role in nutrition and as a source of livelihood, in particular providing employment and income generation for rural and tribal groups (Vijayan et al. 2008, Mikulic-Petkovsek et al. 2012, Ercisli et al. 2012, Cuce and Sokmen 2017). The blue honeysuckle berry (Lonicera caerulea var. edulis Turcz. ex Herder) (WFO 2019) from the Lonicera genus and the plants can be organically grown. Their natural growth areas are wetland spaces along rivers, marshes or forest clearings in northeastern Asia and America (Thompson 2008, Miyashita et al. 2009). The berries are similar in color to blueberries, dark purple with a waxy coating, but with an obvious difference in the berry shape. Blue honeysuckle berries have a more elongated or cylindrical shape than the blueberry (Thompson 2008, Hummer et al. 2012). The taste is bitter to tart-sweet, a mixture of known berry flavors (Hummer et al. 2012). The berries from blue honeysuckle have become popular because of their health-promoting properties. They contain nutraceutical compounds, such as vitamins, minerals, polyphenolics, iridoids and saponins (Jurikova et al. 2009, 2012, Becker et al. 2017, Oszmiański and Kucharska 2018). Additionally, they contain a low sugar content compared to some other fruits, which could make them a good source of nutrition for people with diabetic troubles (Palíková et al. 2009). The berries have a short shelf-life and it is important to keep the product fresh to maintain its nutritional value as far as possible. Most storage techniques require low temperatures, which are difficult to maintain throughout a distribution chain (Sagar and Kumar 2010). In addition to freezing, drying is the simplest

*Corresponding author e-mail: mateja.senica@bf.uni-lj.si 
procedure for preserving fruit (Senica et al. 2016). Not only does it extend the shelf-life of fruit, but it retains the characteristics of natural products, reduces the costs of packing, storage and transportation, due to the reduced weight and volume of the product, and additionally inhibits the growth of micro-organisms (Wang and Xu 2007, Chauhan and Srivastava 2009, Sagar and Kumar 2010, Mundada et al. 2010). Another advantage is the higher prices of commodities and accessibility of food in the season when fresh berries are not available. Disadvantages of drying are an alternation of color, in terms of browning, lipid oxidation, degradation of enzymes and change of aroma, flavour and taste. The texture of dried products is influenced by their moisture content, composition, $\mathrm{pH}$, and product maturity. During drying, the collapse of cell structures causes, reduction in size through shrinkage of the berries (Sagar and Kumar 2010).

Freshly harvested blue honeysuckle berries have a short shelf-life. In Europe, fresh blue honeysuckle berries can be purchased from mid-May to the end of June. There are many food preservation methods for prolonging the presence of fruit in the market and to ensure product nutritional and health quality. The most suitable fruits for drying are apples, pears, plums, grapes, apricots, figs, persimmons and peaches. They can be purchased dried on the market or prepared at home. They are principally dried on average for less than 30 hours (depending on the fruit) at 60 degrees (GardenRobinson 2012, FAO 2019). There have been a several studies investigating anthocyanin contents during different preservation processes in blue honeysuckle berries (Khattab et al. 2016, Oszmiański et al. 2016) and some other fruit species, such as blueberries (Brownmiller et al. 2008, Sablani et al. 2010), persimmon (Karaman et al. 2014, Senica et al. 2016), gooseberry (Kucner et al. 2014) and strawberry (Wojdyło et al. 2009). Mineral content changes were observed in strawberries stored at different temperatures (Çavuşoğlu 2018). The aim of this study was to identify appropriate drying conditions for preparing dried blue honeysuckle berries while achieving a high quality. We evaluated the influence of a combination of different temperatures $(40,50,60,65$ and $\left.75^{\circ} \mathrm{C}\right)$ and times of heating $(240,200,66,30$ and 20 hours) on the thermal stability of ascorbic acid, sugars, organic acids and phenolic content of blue honeysuckle berries.

\section{Materials and methods}

\section{Plant material}

The blue honeysuckle berries were from the cultivar 'Aurora', grown organically in Slovenia. The berries were handharvested at the fully ripe stage on 11 June 2017, at the location Šmartno pri Litiji ( $46^{\circ} 2^{\prime} 38.7^{\prime \prime} \mathrm{N} 14^{\circ} 50^{\prime} 47^{\prime \prime}$ E, $250 \mathrm{~m}$ a.s.l.). Fresh berries were immediately used for control treatment. Other berries were dried in a drying oven at different durations and temperatures. In addition to the control, we applied five treatments: at $40^{\circ} \mathrm{C}$ for 240 hours ( 10 days), at $50^{\circ} \mathrm{C}$ for 200 hours, at $60^{\circ} \mathrm{C}$ at 66 hours, at $65^{\circ} \mathrm{C}$ for 30 hours and at $75^{\circ} \mathrm{C}$ drying for 20 hours. Fifty grams of blue honeysuckle berries were used per repetition (10 repetitions).

\section{Drying process}

The drying process was carried out using a hot-air drying oven (Suša 6, wood dryer, Splošno mizarstvo, Slovenia) at $40,50,60,65$ and $75^{\circ} \mathrm{C}$. Drying was continued for up to $30 \mathrm{~h}$ or the time needed to reach $15 \%$ of moisture or lower.

\section{Determination of ascorbic acid in dried honeysuckle berries}

For control $5 \mathrm{~g}$ of berries mashed and extracted with $10 \mathrm{ml}$ of $2 \%$ meta-phosphoric acid was used. Half of one gram of dried berries from each drying treatment was extracted with $5 \mathrm{ml}$ of $2 \%$ meta-phosphoric acid. The control and all dried mixtures were then left at room temperature for 1 hour on a shaker (Grant-Bio POS-300, Grant Instruments, Shepreth, England) for ascorbic acid extraction. The samples were afterward centrifuged at $4^{\circ} \mathrm{C}$ at $10000 \mathrm{rpm}$ for 7 min and filtered through a Chromafil A-20/25 mixed ester filter (Macherey-Nagel, Düren, Germany) into vials and left to wait until HPLC analysis. Determination of ascorbic acid was carried out with the Thermo Finnigan Surveyor HPLC system (Thermo Scientific, San Jose, CA). Conditions were previously described in Mikulic-Petkovsek et al. (2016) study. Contents were expressed in mg of ascorbic acid per $100 \mathrm{~g}$ of dry weight.

\section{Determination of sugars and organic acids in dried honeysuckle berries}

Sugar and organic acid contents among various dried honeysuckle berries were estimated according to the method of Mikulic-Petkovsek et al. (2016). For control $5 \mathrm{~g}$ of fresh honeysuckle berries was mixed with an Ultra-Turrax T-25 macerator and extracted with $25 \mathrm{ml}$ double-distilled water. Half of one gram of dried honeysuckle berries was extracted with $10 \mathrm{ml}$ of double-distilled water. Each sample was then left at room temperature for 1 hour on a shaker. Samples were afterwards centrifuged at $4{ }^{\circ} \mathrm{C}$ at $10000 \mathrm{rpm}$ for $7 \mathrm{~min}$ and filtered through a Chromafil A-20/25 mixed ester filter into vials and allowed to wait until HPLC analysis. Determination of individual sugars and organic acids was carried out with the Thermo Finnigan Surveyor HPLC system, as previously described in Mikulic-Petkovsek et al. (2016) study. Contents were expressed in mg per g of dry weight.

\section{Determination of individual phenolics}

The extraction of phenolic compounds for 5 different honeysuckle berry products was carried out as described by Senica et al. (2016) with some modifications. For control honeysuckle berries were homogenized with an Ultra-Turrax T-25 and $5 \mathrm{~g}$ of fruit paste was extracted in $30 \mathrm{ml}$-centrifuge tubes with $15 \mathrm{ml}$ methanol containing 3\% formic acid. For other treatments, one gram of dried honeysuckle berries was extracted with $10 \mathrm{ml}$ of methanol containing $3 \%$ formic acid. All samples were then placed in a cool ultrasonic bath for 1 hour. The mixtures were then centrifuged for $10 \mathrm{~min}$ at $12000 \mathrm{rpm}$. Each supernatant was filtered through a Chromafil AO-20/25 polyamide filter (Macherey-Nagel, Düren, Germany) and transferred into vials until HPLC and MS 
analysis. Separation of phenolic compounds was performed on a mass spectrometer (LCQ Deca XP MAX, Thermo Scientific) with electrospray ionization (ESI) operated in negative and positive ion modes. The ESI parameters were described by Senica et al. (2016). Analyses were carried out using the Accela HPLC system (Thermo Scientific, San Jose, CA), equipped with a diode array detector (DAD), controlled by CromQuest 4.0 chromatography workstation software, with technical characteristics as described by Senica et al. (2016) with the mobile phase gradient according to Wang et al. (2002). Individual phenolic compounds were identified by fragmentation with HPLC-MS, comparison of retention times with standards and monitoring UV-VIS spectra from $200-550 \mathrm{~nm}$. Calibration curves were prepared from all standards and the individual compounds were identified and quantified by comparison with pure standards.

\section{Chemicals}

Quinic acid, shikimic acid, 5-caffeoylquinic acid, neochlorogenic acid 3-caffeoylqinic acid, cyanidin-3-glucoside, ellagic acid, naringenin and luteolin-3-rutinoside and ascorbic acid standards as well as meta-phosphoric acid and methanol were obtained from Sigma Aldrich Chemie (Steinheim, Germany). We obtained fructose, glucose, sucrose, citric, malic, fumaric and tartatic acid, standards for sugars and organic acids; additionally epicatechin, quercetin-3-galactoside, quercetin-3-glucoside, quercetin-3-rutinoside, $p$-coumaric acid, procyanidin B2, luteolin-3-glucoside, genistein and kaempferol-3-glucoside for standards of phenolics from Fluka Chemie (Buchs, Switzerland). Phenolic standards catechin, $p$-coumaric acid and caffeic acid were obtained from Roth (Karlsruhe, Germany); quercetin-3-xyloside, quercetin-3-arabinofuranoside from Apin Chemicals (Abingdon, UK) and isorhamnetin-3-rutinoside, loganin, petunidin- and peonidin-3-glucoside from Extrasynthese (Genay, Frence). Ultrapure water used to prepare all water extractions and the mobile phases was obtained from the Milli-Q system (Millipore, Bedford, MA, USA). For phenolics where standards were lacking, they were tentatively identified based on their fragmentation pattern obtained from $\mathrm{MS}^{2} / \mathrm{MS}^{3}$ analysis and by comparison with data from the literature. Their contents were calculated using chemically similar phenolic compounds. Thus quercetin glycosides were quantified in equivalents of quercetin-3-galactoside, isorhamnetin glycosides in equivalents of isorhamnetin-3-rutinoside, luteolin derivatives in equivalents of luteolin-3-glucoside, genistein derivate in equivalents of genistein, kaempferol glycosides in equivalents of kaempferol-3-glucoside, procyanidins in equivalents of procyanidin $\mathrm{B} 2$, pelargonidin- and peonidin derivatives on pelargonidin or peonidin-3-glucoside and ellagic acid derivatives in equivalents of ellagic acid.

\section{Statistical analysis}

The results were analyzed statistically using a one way analysis of variance (ANOVA) with the statistical program $\mathrm{R}$ commander. Duncan's mean separation tests were done for comparisons of the contents of the primary and secondary metabolites studied. Statistically significant differences were accepted at $\mathrm{P}<0.05$.

\section{Results}

All compounds were expressed on a dry weight basis to ensure reliable comparison among different thermal treatments. At lower temperatures, they needed a longer time to dry and for water to be removed from the fruit. The contents of ascorbic and organic acids as well as of sugars in dried honeysuckle berries are shown in Tab. 1. In Tab. 2, the identification of individual phenolic compounds is given, while their contents are presented in Tab. 3.

\section{Sugars, ascorbic and organic acid levels}

Contents of sugars, glucose, fructose and sucrose in blue honeysuckle berry fruit were determined. Fructose content ranged from 51 to $58 \%$, glucose from 41 to $47 \%$ and sucrose from $1-5 \%$ of total sugar content (Tab. 1). Determined fruc-

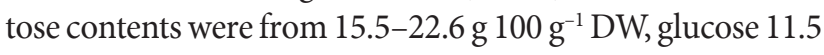

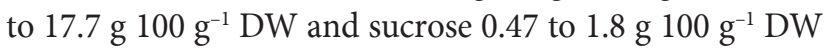
of honeysuckle berries. In general, sugar contents in dried berries dropped one third less than non-treated berries. The highest total sugar content was measured in honeysuckle

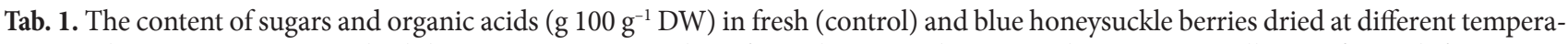
tures and times. Means \pm standard deviation are presented. Different letters (a-d) in rows denote statistically significant differences in some primary metabolites among fresh and dried blue honeysuckle berries by Duncan multiple range test $(\mathrm{P}<0.05) ; \mathrm{n}=10$.

\begin{tabular}{lcccccc}
\hline Parameters & Control & $40{ }^{\circ} \mathrm{C}(240 \mathrm{~h})$ & $50^{\circ} \mathrm{C}(200 \mathrm{~h})$ & $60^{\circ} \mathrm{C}(66 \mathrm{~h})$ & $65^{\circ} \mathrm{C}(30 \mathrm{~h})$ & $75^{\circ} \mathrm{C}(20 \mathrm{~h})$ \\
\hline Fructose & $22.57 \pm 0.51^{\mathrm{a}}$ & $15.58 \pm 1.37^{\mathrm{c}}$ & $17.5 \pm 0.87^{\mathrm{b}}$ & $17.21 \pm 1.13^{\mathrm{b}}$ & $17.83 \pm 0.41^{\mathrm{b}}$ & $15.96 \pm 0.63^{\mathrm{c}}$ \\
Glucose & $17.75 \pm 0.28^{\mathrm{a}}$ & $14.23 \pm 0.98^{\mathrm{c}}$ & $15.29 \pm 0.72^{\mathrm{b}}$ & $12.82 \pm 0.99^{\mathrm{d}}$ & $13.24 \pm 0.45^{\mathrm{d}}$ & $11.50 \pm 0.54^{\mathrm{e}}$ \\
Sucrose & $0.86 \pm 0.21^{\mathrm{c}}$ & $0.65 \pm 0.22^{\mathrm{c}}$ & $0.47 \pm 0.31^{\mathrm{cd}}$ & $1.40 \pm 0.52^{\mathrm{b}}$ & $1.78 \pm 0.22^{\mathrm{a}}$ & $0.18 \pm 0.04^{\mathrm{d}}$ \\
\hline Citric acid & $18.10 \pm 1.30^{\mathrm{a}}$ & $6.28 \pm 0.54^{\mathrm{f}}$ & $9.74 \pm 1.16^{\mathrm{d}}$ & $8.44 \pm 1.07^{\mathrm{e}}$ & $15.14 \pm 0.50^{\mathrm{b}}$ & $11.67 \pm 0.30^{\mathrm{c}}$ \\
Fumaric acid & $0.002 \pm 0.00^{\mathrm{d}}$ & $0.009 \pm 0.002^{\mathrm{c}}$ & $0.010 \pm 0.001^{\mathrm{bc}}$ & $0.003 \pm 0.001^{\mathrm{d}}$ & $0.013 \pm 0.001^{\mathrm{a}}$ & $0.012 \pm 0.001^{\mathrm{b}}$ \\
Malic acid & $5.91 \pm 0.28^{\mathrm{b}}$ & $3.71 \pm 0.40^{\mathrm{c}}$ & $5.37 \pm 0.66^{\mathrm{b}}$ & $2.63 \pm 0.46^{\mathrm{d}}$ & $7.79 \pm 0.53^{\mathrm{a}}$ & $7.82 \pm 0.28^{\mathrm{a}}$ \\
Shikimic acid & $0.002 \pm 0.001^{\mathrm{c}}$ & $0.022 \pm 0.007^{\mathrm{b}}$ & $0.038 \pm 0.008^{\mathrm{a}}$ & $0.004 \pm 0.001^{\mathrm{c}}$ & $0.019 \pm 0.002^{\mathrm{b}}$ & $0.023 \pm 0.001^{\mathrm{b}}$ \\
Tartaric acid & $1.23 \pm 0.12^{\mathrm{d}}$ & $2.49 \pm 0.52^{\mathrm{c}}$ & $3.54 \pm 0.61^{\mathrm{b}}$ & $1.38 \pm 0.05^{\mathrm{d}}$ & $4.61 \pm 0.38^{\mathrm{a}}$ & $4.37 \pm 0.10^{\mathrm{a}}$ \\
Quinic acid & $6.62 \pm 0.26^{\mathrm{a}}$ & $4.76 \pm 0.42^{\mathrm{b}}$ & $6.10 \pm 0.78^{\mathrm{ab}}$ & $3.16 \pm 0.33^{\mathrm{c}}$ & $6.56 \pm 0.46^{\mathrm{a}}$ & $7.44 \pm 0.92^{\mathrm{a}}$ \\
\hline
\end{tabular}


Tab. 2. Identification of phenolic compounds in blue honeysuckle fruits in positive and negative ions with HPLC-MS, MS ${ }^{2}$ and $\mathrm{MS}^{3}$.

\begin{tabular}{|c|c|c|c|c|}
\hline Phenolic group & {$[\mathrm{M}]^{+}$or $[\mathrm{M}-\mathrm{H}]^{-}(\mathrm{m} / \mathrm{z})$} & $\mathrm{MS}^{2}(\mathrm{~m} / \mathrm{z})$ & $\mathrm{MS}^{3}(\mathrm{~m} / \mathrm{z})$ & Phenolic compound \\
\hline \multirow[t]{6}{*}{ Hydroxycinnamic acid } & 353 & $191,179,135$ & & Neochlorogenic acid (3-caffeoylquinic acid) \\
\hline & 353 & $173,179,191$ & & Cryptochlorogenic acid (4-caffeoylquinic acid) \\
\hline & 353 & $191,179,173,135$ & & Chlorogenic acid (5-caffeoylquinic acid) \\
\hline & 337 & $191,173,163$ & & 5-Coumaroylquinic acid \\
\hline & 325 & 163,119 & & $p$-coumaric acid hexoside \\
\hline & 515 & 353 & $191,179,173$ & Dicaffeoylquinic acid \\
\hline Hydroxybenzoic acids & 463 & 301 & 257,229 & Ellagic acid hexoside \\
\hline \multirow[t]{4}{*}{ Flavanols } & 289 & 245 & & Catechin \\
\hline & 289 & 245 & & Epicatechin \\
\hline & 577 & $425,407,289$ & & Procyanidin dimer \\
\hline & 865 & $577,451,425,407,289$ & & Procyanidin trimer \\
\hline \multirow[t]{2}{*}{ Flavones } & 447 & 285 & & Luteolin hexoside \\
\hline & 593 & 447 & 285 & Luteolin-3-rutinoside \\
\hline Isoflavones & 449 & 269 & & Genistein hydroxyhexoside \\
\hline \multirow[t]{17}{*}{ Flavonols } & 519 & 315 & & Isorhamnetin acetyhexoside \\
\hline & 665 & 315 & & Isorhamnetin acetyl rhamnosylhexoside \\
\hline & 609 & 315 & & Isorhamnetin hexosylpentoside \\
\hline & 623 & 315 & & Isorhamnetin-3-rutinoside \\
\hline & 489 & 285 & & Kaempferol acetylhexoside \\
\hline & 579 & 285 & & Kaempferol hexosylpentoside \\
\hline & 593 & 285 & & Kaempferol-3-rutinoside \\
\hline & 447 & 285 & & Kaempferol-3-glucoside \\
\hline & 505 & 301 & & Quercetin-3-acetylhexoside \\
\hline & 433 & 301 & & Quercetin-3-arabinofuranoside \\
\hline & 463 & 301 & & Quercetin-3-galactoside \\
\hline & 463 & 301 & & Quercetin-3-glucoside \\
\hline & 463 & 301 & & Quercetin hexoside \\
\hline & 595 & 301 & & Quercetin hexoside pentoside \\
\hline & 609 & 301 & & Quercetin-3-rutinoside \\
\hline & 595 & 301 & & Quercetin-3-vicianoside \\
\hline & 433 & 301 & & Quercetin-3-xyloside \\
\hline Flavanones & 433 & 271 & & Naringenin hexoside \\
\hline Iridoid & 521 & 389,227 & & Loganin-7-pentoside \\
\hline \multirow[t]{7}{*}{ Anthocyanins } & 611 & $449 / 287$ & & Cyanidin-3,5-diglucoside \\
\hline & 449 & 287 & & Cyanidin-3-glucoside \\
\hline & 595 & $449 / 287$ & & Cyanidin-3-rutinoside \\
\hline & 595 & $433 / 271$ & & Pelargonidin dihexoside \\
\hline & 433 & 271 & & Pelargonidin-3-glucoside \\
\hline & 625 & $463 / 301$ & & Peonidin dihexoside \\
\hline & 463 & 301 & & Peonidin-3-glucoside \\
\hline
\end{tabular}

berries dried at $50{ }^{\circ} \mathrm{C}$ for 200 hours and at $65^{\circ} \mathrm{C}$ for 30 hours, after which sugar contents were $20 \%$ lower than before drying (Fig. 1). The lowest total sugar content was measured in honeysuckle berries dried at $75^{\circ} \mathrm{C}$ for 20 hours, with their contents $33 \%$ lower than in the control.

The drying process caused a decrease of ascorbic acid as well. Fresh berries contained $154.89 \mathrm{mg} 100 \mathrm{~g}^{-1} \mathrm{DW}$ of ascorbic acid. After the heat treatment there was no detected vitamin $\mathrm{C}$ content in any dried blue honeysuckle berries.

Five organic acids were identified in the blue honeysuckle berries. The most abundant was citric acid (36 to $57 \%$ of total organic acids), followed by malic and quinic acids (19 to $28 \%$ ), tartaric acid (4 to $14 \%$ ), while fumaric and shikimic acids contributed under $1 \%$ of total organic acids (Tab. 1).
In general, the contents of organic acids significantly varied according to the different heat treatments (Fig. 1), but all organic acids slightly increased (Tab. 1) with heating above $65^{\circ} \mathrm{C}$ (Fig. 2).

\section{Phenolic compounds composition}

Forty different individual phenolics were quantified by HPLC-MS in the honeysuckle berries (Tab. 2). We detected and identified some less known phenolics, such as loganinpentoside, an iridoid determined according to its molecular ion at $m / z 521[\mathrm{M}-\mathrm{H}]^{-}$and its corresponding fragment ions $m / z 389,227$. An isoflavone genistein hydroxyhexoside (Tab. 2) was confirmed according to the fragmentation pattern; from molecular ion at $m / z 449$ we got fragment ion 


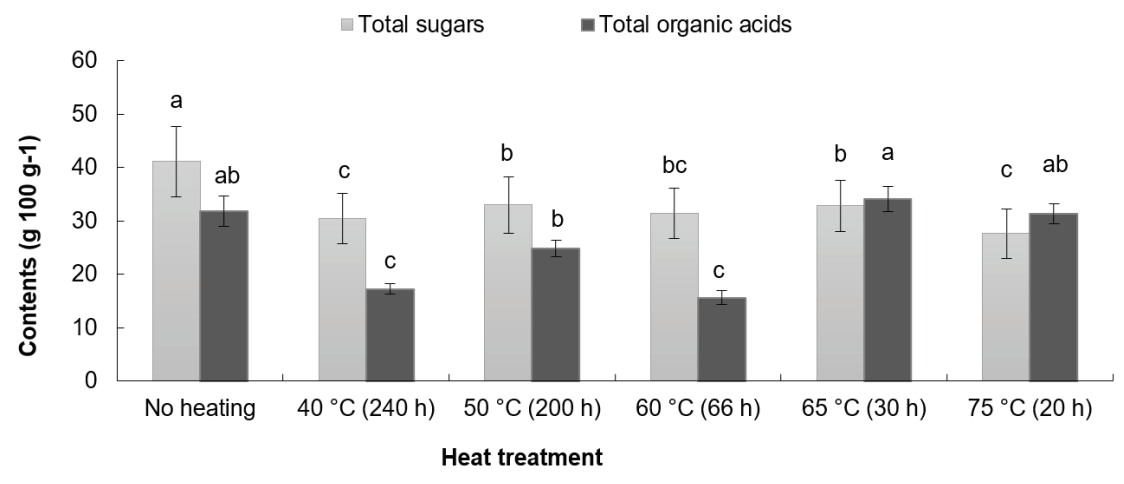

Fig. 1. The content of total sugars and organic acids during the different heating treatments expressed per $100 \mathrm{~g}$ DW. Different letters (a-d) mean significant differences among different heat treatments $(\mathrm{P}<0.05)$ by Duncan's multiple range test.

$m / z$ 269. Ellagic acid hexoside was identified according to its molecular ion at $m / z 463[\mathrm{M}-\mathrm{H}]^{-}$and its corresponding fragment ions $m / z 301,229$ and 257. All phenolic compounds were divided into 9 groups (Tab. 2). The groups in our study had different tolerances to temperature and time of heating (Fig. 2).

The group of hydroxycinnamic acids (HCA) represented only $3 \%$ of total phenolics in fresh blue honeysuckle berries. The main contributors to HCA in fresh berries were neochlorogenic (3-caffeoylquinic acid) and dicaffeoylquinic acids, while in dried blue honeysuckle berries there were neochlorogenic and $p$-coumaric acids (Tab. 3). In our study their content was higher after the drying than in fresh berries. The highest content of total HCA derivatives was measured in honeysuckle berries dried at $65^{\circ} \mathrm{C}$ for $30 \mathrm{~h}(355.94$ mg $100 \mathrm{~g}^{-1}$ ) (Fig. 2).

Flavanols (catechin, epicatechin and procyanidins) contribute approximately $50 \%$ of total analyzed phenolics in fresh berries. Their contents decreased with heating. The highest level, which was still only half that of fresh berries, was determined in berries dried at $60^{\circ} \mathrm{C}$ for $66 \mathrm{~h}(374.13 \mathrm{mg}$ $\left.100 \mathrm{~g}^{-1}\right)$. Berries dried at $50^{\circ} \mathrm{C}$ for $200 \mathrm{~h}\left(190.45 \mathrm{mg} 100 \mathrm{~g}^{-1}\right.$ DW) had the lowest flavanol content (Tab. 3).
The group of flavonols (quercetin, kaempferol and isorhamnetin glycosides), with up to $18 \%$ of total analyzed phenolics, did not show a clear trend of increase or decrease with heating. At temperatures of $60^{\circ} \mathrm{C}$ and $75^{\circ} \mathrm{C}$, the content of flavonols was higher than in the control, while their contents in other treatments slightly decreased (Fig. 2). Honeysuckle berries dried at $75^{\circ} \mathrm{C}$ for $20 \mathrm{~h}$ had the highest flavonol content $\left(326.60 \mathrm{mg} 100 \mathrm{~g}^{-1}\right)$, while berries dried at $65^{\circ} \mathrm{C}$ for $30 \mathrm{~h}$ had the lowest flavonol content (248.61 mg $100 \mathrm{~g}^{-1}$ ) (Tab. 3).

Flavanones and flavones contributed less than $1 \%$ of total phenolics. Naringenin hexoside of the flavanone group showed a decrease in content with longer heating time and an increase at higher temperatures. Flavones in our study decreased with all heat treatments, but a higher decrease occurred after longer drying than at higher temperature (Tab. 3).

In our study, anthocyanins comprising $26 \%$ of the total phenolics, had the lowest content among phenolics in all heat treatments (Fig. 2). The most abundant of total anthocyanins was cyanidin-3-glucoside and the least was pelargonidin-dihexoside. Cyanidin-3-glucoside represented from 45 to $65 \%$ of total anthocyanins in the various drying treatments. Their level decreased by $64 \%$ with drying at $40{ }^{\circ} \mathrm{C}$ for 240 hours, by $80 \%$ at $50{ }^{\circ} \mathrm{C}$ for 200 hours, by $57 \%$ at $60^{\circ} \mathrm{C}$

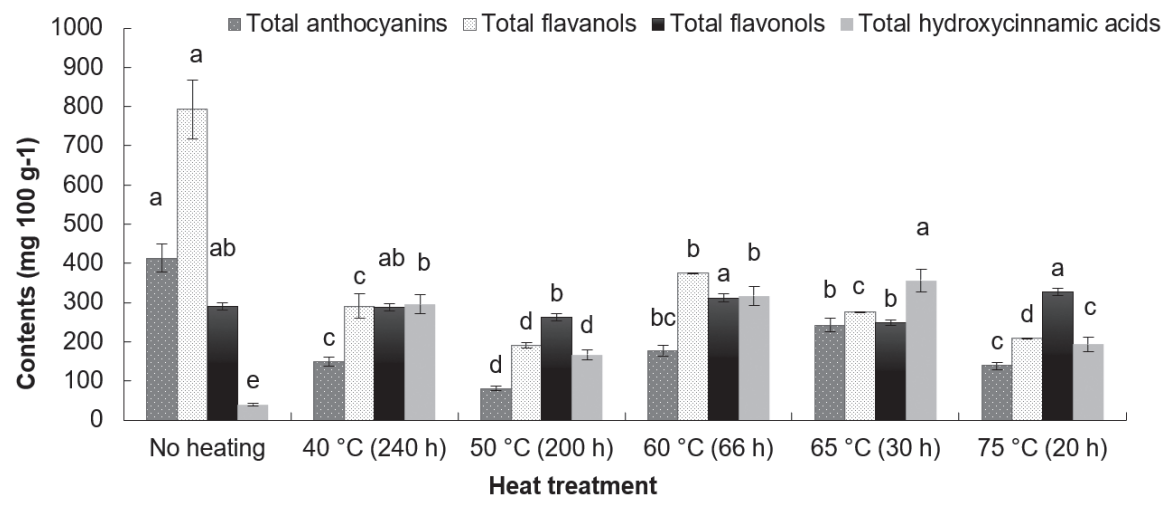

Fig. 2. The content of different phenolic groups during the different heating treatments expressed per $100 \mathrm{~g}$ DW. Different letters (a-d) mean significant differences among different heat treatments $(\mathrm{P}<0.05)$ by Duncan's multiple range test. 
Tab. 3. Individual phenolics content ( $\mathrm{mg} 100 \mathrm{~g}^{-1} \mathrm{DW}$ ) in six different blue honeysuckle berry products. Means \pm standard deviation are presented. Different letters (a-f) in rows denote statistically significant differences in individual phenolic levels among blue honeysuckle berry products by Duncan's multiple range test $(\mathrm{P}<0.05) ; \mathrm{n}=10$.

\begin{tabular}{|c|c|c|c|c|c|c|}
\hline & Control & $\begin{array}{l}40^{\circ} \mathrm{C} \\
(240 \mathrm{~h})\end{array}$ & $\begin{array}{l}50^{\circ} \mathrm{C} \\
(200 \mathrm{~h})\end{array}$ & $\begin{array}{l}60^{\circ} \mathrm{C} \\
(66 \mathrm{~h})\end{array}$ & $\begin{array}{l}65^{\circ} \mathrm{C} \\
(30 \mathrm{~h})\end{array}$ & $\begin{array}{l}75^{\circ} \mathrm{C} \\
(20 \mathrm{~h})\end{array}$ \\
\hline \multicolumn{7}{|l|}{ Hydroxycinamic acids } \\
\hline $\begin{array}{l}\text { Neochlorogenic acid } \\
\text { (3-CQA) }\end{array}$ & $19.69 \pm 0.34^{\mathrm{e}}$ & $154.56 \pm 8.97^{\mathrm{b}}$ & $86.70 \pm 3.56^{\mathrm{d}}$ & $161.58 \pm 20.94^{\mathrm{b}}$ & $198.99 \pm 15.14^{\mathrm{a}}$ & $119.10 \pm 23.42^{c}$ \\
\hline $\begin{array}{l}\text { Cryptochlorogenic acid } \\
\text { (4-CQA) }\end{array}$ & $0.57 \pm 0.01^{\mathrm{c}}$ & $56.22 \pm 5.72^{\mathrm{a}}$ & $32.78 \pm 3.54^{\mathrm{b}}$ & $57.30 \pm 8.80^{\mathrm{a}}$ & $54.35 \pm 3.61^{\mathrm{a}}$ & $40.34 \pm 8.92^{\mathrm{b}}$ \\
\hline Chlorogenic acid (5-CQA) & $0.26 \pm 0.01^{\mathrm{a}}$ & $0.04 \pm 0.00^{\mathrm{d}}$ & $0.02 \pm 0.00^{\mathrm{e}}$ & $0.05 \pm 0.00^{c}$ & $0.07 \pm 0.00^{\mathrm{b}}$ & $0.01 \pm 0.00^{\mathrm{f}}$ \\
\hline Coumaroylquinic acid & $1.84 \pm 0.01^{\mathrm{e}}$ & $5.87 \pm 0.55^{\mathrm{d}}$ & $9.21 \pm 0.46^{\mathrm{c}}$ & $34.56 \pm 0.70^{\mathrm{a}}$ & $19.75 \pm 1.10^{\mathrm{b}}$ & $6.72 \pm 0.75^{\mathrm{d}}$ \\
\hline$p$-Coumaric acid hexoside & $2.99 \pm 0.27^{\mathrm{d}}$ & $68.05 \pm 5.65^{\mathrm{a}}$ & $30.26 \pm 3.78^{\mathrm{bc}}$ & $48.90 \pm 4.65^{\mathrm{ab}}$ & $69.99 \pm 2.81^{\mathrm{a}}$ & $14.96 \pm 1.84^{\text {cd }}$ \\
\hline Dicaffeoylquinic acid & $14.13 \pm 0.39^{\mathrm{a}}$ & $10.51 \pm 0.73^{\mathrm{c}}$ & $7.48 \pm 0.40^{\mathrm{d}}$ & $14.38 \pm 0.56^{\mathrm{a}}$ & $12.79 \pm 1.34^{\mathrm{b}}$ & $11.77 \pm 1.71^{\mathrm{bc}}$ \\
\hline \multicolumn{7}{|l|}{ Hhydroxybenzoic acids } \\
\hline Ellagic acid hexoside & $16.09 \pm 1.75^{\mathrm{b}}$ & $1.6 \pm 0.27^{\mathrm{e}}$ & $7.12 \pm 0.41^{\mathrm{d}}$ & $9.40 \pm 1.03^{\mathrm{c}}$ & $10.73 \pm 0.72^{\mathrm{c}}$ & $23.44 \pm 1.28^{\mathrm{a}}$ \\
\hline \multicolumn{7}{|l|}{ Flavanols } \\
\hline$(+)$ catechin & $20.04 \pm 2.76^{\mathrm{a}}$ & $16.03 \pm 1.31^{\mathrm{bc}}$ & $7.93 \pm 0.63^{\mathrm{e}}$ & $17.21 \pm 1.36^{\mathrm{b}}$ & $14.59 \pm 1.11^{\mathrm{c}}$ & $10.05 \pm 0.79^{\mathrm{d}}$ \\
\hline (-)epicatechin & $232.83 \pm 23.50^{\mathrm{a}}$ & $89.40 \pm 8.11^{\mathrm{c}}$ & $81.62 \pm 14.64^{\mathrm{c}}$ & $142.13 \pm 18.31^{\mathrm{b}}$ & $91.77 \pm 6.70^{c}$ & $75.25 \pm 2.55^{\mathrm{c}}$ \\
\hline Procyanidin dimer & $539.20 \pm 40.48^{\mathrm{a}}$ & $184.93 \pm 18.17^{c}$ & $100.73 \pm 14.99^{\mathrm{d}}$ & $214.37 \pm 11.93^{\mathrm{b}}$ & $169.72 \pm 6.68^{c}$ & $122.70 \pm 10.15^{\mathrm{d}}$ \\
\hline Procyanidin trimer & $0.55 \pm 0.05^{\mathrm{b}}$ & $0.49 \pm 0.03^{\mathrm{bc}}$ & $0.16 \pm 0.02^{\mathrm{d}}$ & $0.42 \pm 0.03^{\mathrm{c}}$ & $0.21 \pm 0.03^{\mathrm{d}}$ & $1.13 \pm 0.15^{\mathrm{a}}$ \\
\hline \multicolumn{7}{|l|}{ Flavones } \\
\hline Luteolin hexoside & $3.01 \pm 0.15^{\mathrm{a}}$ & $1.55 \pm 0.81^{\mathrm{cd}}$ & $1.13 \pm 0.09^{\mathrm{d}}$ & $1.83 \pm 0.11^{\mathrm{bc}}$ & $1.49 \pm 0.12^{\mathrm{cd}}$ & $2.09 \pm 0.31^{\mathrm{b}}$ \\
\hline Luteolin-3-rutinoside & $4.85 \pm 0.21^{\mathrm{a}}$ & $3.84 \pm 0.65^{\mathrm{b}}$ & $4.56 \pm 0.47^{\mathrm{a}}$ & $4.81 \pm 0.48^{\mathrm{a}}$ & $2.05 \pm 0.15^{\mathrm{c}}$ & $4.56 \pm 0.51^{\mathrm{a}}$ \\
\hline \multicolumn{7}{|l|}{ Isoflavone } \\
\hline Genistein hydroxyhexoside & $1.91 \pm 0.07^{\mathrm{a}}$ & $1.05 \pm 0.01^{\mathrm{d}}$ & $1.05 \pm 0.01^{\mathrm{d}}$ & $1.30 \pm 0.10^{\mathrm{c}}$ & $2.07 \pm 0.19^{\mathrm{a}}$ & $1.53 \pm 0.07^{\mathrm{b}}$ \\
\hline \multicolumn{7}{|l|}{ Flavonols } \\
\hline Isorhamnetin acetylhexoside & $1.47 \pm 0.16^{\mathrm{a}}$ & $0.03 \pm 0.00^{\mathrm{b}}$ & $0.01 \pm 0.00^{\mathrm{b}}$ & $0.02 \pm 0.00^{\mathrm{b}}$ & $0.01 \pm 0.00^{\mathrm{b}}$ & $0.02 \pm 0.00^{\mathrm{b}}$ \\
\hline $\begin{array}{l}\text { Isorhamnetin acetyl } \\
\text { rhamnosyl hexoside }\end{array}$ & $10.91 \pm 0.61^{\mathrm{ab}}$ & $10.47 \pm 1.31^{\mathrm{ab}}$ & $6.31 \pm 0.38^{\mathrm{c}}$ & $11.78 \pm 0.56^{\mathrm{a}}$ & $10.26 \pm 1.06^{\mathrm{b}}$ & $9.69 \pm 1.40^{\mathrm{b}}$ \\
\hline $\begin{array}{l}\text { Isorhamnetin } \\
\text { hexosylpentoside }\end{array}$ & $1.95 \pm 0.11^{\mathrm{d}}$ & $4.49 \pm 0.86^{c}$ & $5.55 \pm 0.53^{\mathrm{b}}$ & $6.20 \pm 0.29^{\mathrm{a}}$ & $5.28 \pm 0.44^{\mathrm{b}}$ & $6.35 \pm 0.10^{\mathrm{a}}$ \\
\hline Isorhamnetin-3-rutinoside & $14.08 \pm 0.92^{\mathrm{a}}$ & $9.21 \pm 0.97^{c}$ & $9.90 \pm 1.64^{\mathrm{c}}$ & $12.99 \pm 0.44^{\mathrm{ab}}$ & $9.32 \pm 1.17^{\mathrm{c}}$ & $11.98 \pm 1.27^{\mathrm{b}}$ \\
\hline Kaempferol acetylhexoside & $0.51 \pm 0.00^{\mathrm{d}}$ & $1.30 \pm 0.16^{\mathrm{a}}$ & $0.47 \pm 0.03^{\mathrm{d}}$ & $0.97 \pm 0.03^{\mathrm{b}}$ & $0.74 \pm 0.05^{\mathrm{c}}$ & $0.89 \pm 0.09^{\mathrm{b}}$ \\
\hline $\begin{array}{l}\text { Kaempferol } \\
\text { hexosylpentoside }\end{array}$ & $3.55 \pm 0.08^{\mathrm{a}}$ & $1.23 \pm 1.72^{\mathrm{b}}$ & $0.58 \pm 0.05^{\mathrm{b}}$ & $0.70 \pm 0.03^{\mathrm{b}}$ & $0.75 \pm 0.05^{\mathrm{b}}$ & $1.01 \pm 0.16^{\mathrm{b}}$ \\
\hline Kaempferol-3-rutinoside & $2.20 \pm 0.25^{\mathrm{e}}$ & $2.53 \pm 0.29^{\mathrm{de}}$ & $3.03 \pm 0.37^{\mathrm{cd}}$ & $3.56 \pm 0.34^{\mathrm{bc}}$ & $3.88 \pm 0.58^{\mathrm{b}}$ & $5.47 \pm 0.86^{\mathrm{a}}$ \\
\hline Kaempferol-3-glucoside & $0.06 \pm 0.00^{\mathrm{a}}$ & $0.10 \pm 0.01^{\mathrm{a}}$ & $0.15 \pm 0.02^{\mathrm{a}}$ & $0.12 \pm 0.01^{\mathrm{a}}$ & $0.09 \pm 0.01^{\mathrm{a}}$ & $0.09 \pm 0.01^{\mathrm{a}}$ \\
\hline Quercetin-3-acetylhexoside & $1.95 \pm 0.12^{\mathrm{e}}$ & $3.09 \pm 0.06^{\mathrm{b}}$ & $2.54 \pm 0.51^{\mathrm{d}}$ & $3.53 \pm 0.22^{\mathrm{a}}$ & $3.04 \pm 0.28^{\mathrm{bc}}$ & $2.64 \pm 0.44^{\mathrm{cd}}$ \\
\hline $\begin{array}{l}\text { Quercetin-3- } \\
\text { arabinofuranoside }\end{array}$ & $18.90 \pm 0.96^{\mathrm{a}}$ & $7.68 \pm 0.99^{c}$ & $4.88 \pm 0.28^{\mathrm{d}}$ & $9.46 \pm 0.38^{\mathrm{b}}$ & $7.82 \pm 0.63^{\mathrm{c}}$ & $7.15 \pm 0.70^{c}$ \\
\hline Quercetin-3-galactoside & $30.73 \pm 1.90^{\mathrm{d}}$ & $30.43 \pm 2.66^{\mathrm{d}}$ & $23.90 \pm 3.05^{\mathrm{e}}$ & $43.93 \pm 2.68^{\mathrm{b}}$ & $35.98 \pm 2.97^{c}$ & $54.52 \pm 2.97^{\mathrm{a}}$ \\
\hline Quercetin-3-glucoside & $4.29 \pm 0.12^{\mathrm{d}}$ & $37.74 \pm 8.85^{\mathrm{a}}$ & $30.38 \pm 4.40^{\mathrm{bc}}$ & $34.06 \pm 3.37^{\mathrm{ab}}$ & $24.20 \pm 1.92^{\mathrm{c}}$ & $37.95 \pm 4.90^{\mathrm{a}}$ \\
\hline Quercetin-hexoside & $2.33 \pm 0.19^{\mathrm{a}}$ & $0.57 \pm 0.08^{\mathrm{d}}$ & $0.15 \pm 0.03^{\mathrm{e}}$ & $0.76 \pm 0.09^{c}$ & $2.37 \pm 0.14^{\mathrm{a}}$ & $1.92 \pm 0.24^{\mathrm{b}}$ \\
\hline Q hexoside-pentoside & $6.60 \pm 0.05^{\mathrm{a}}$ & $3.11 \pm 0.82^{\mathrm{d}}$ & $3.89 \pm 0.65^{\mathrm{c}}$ & $3.88 \pm 0.31^{\mathrm{c}}$ & $3.49 \pm 0.32^{\mathrm{cd}}$ & $4.85 \pm 0.18^{\mathrm{b}}$ \\
\hline Quercetin-3-rutinoside & $173.44 \pm 15.19^{\mathrm{a}}$ & $163.47 \pm 16.10^{\mathrm{a}}$ & $157.39 \pm 17.29^{\mathrm{a}}$ & $165.13 \pm 11.90^{\mathrm{a}}$ & $131.02 \pm 12.15^{\mathrm{b}}$ & $167.08 \pm 14.52^{\mathrm{a}}$ \\
\hline Quercetin-3-vicianoside & $16.24 \pm 0.55^{\mathrm{a}}$ & $11.89 \pm 0.81^{\mathrm{cd}}$ & $12.45 \pm 2.42^{\mathrm{bc}}$ & $14.11 \pm 1.72^{\mathrm{ab}}$ & $10.02 \pm 0.91^{\mathrm{d}}$ & $14.56 \pm 2.09^{\mathrm{ab}}$ \\
\hline Quercetin-3-xyloside & $0.40 \pm 0.03^{\mathrm{ab}}$ & $0.32 \pm 0.03^{c}$ & $0.36 \pm 0.06^{\mathrm{bc}}$ & $0.45 \pm 0.05^{\mathrm{a}}$ & $0.34 \pm 0.04^{c}$ & $0.44 \pm 0.05^{\mathrm{a}}$ \\
\hline \multicolumn{7}{|l|}{ Flavanones } \\
\hline Naringenin hexoside & $1.36 \pm 0.04^{c}$ & $0.76 \pm 0.13^{\mathrm{e}}$ & $0.98 \pm 0.09^{\mathrm{de}}$ & $1.72 \pm 0.11^{\mathrm{b}}$ & $1.19 \pm 0.14^{\mathrm{cd}}$ & $2.27 \pm 0.37^{\mathrm{a}}$ \\
\hline \multicolumn{7}{|l|}{ Iridoid } \\
\hline Loganin-7-pentoside & $3.28 \pm 0.38^{\mathrm{b}}$ & $4.23 \pm 0.75^{\mathrm{a}}$ & $1.81 \pm 0.16^{\mathrm{c}}$ & $2.06 \pm 0.10^{c}$ & $1.80 \pm 0.31^{\mathrm{c}}$ & $0.99 \pm 0.03^{\mathrm{d}}$ \\
\hline \multicolumn{7}{|l|}{ Anthocyanins } \\
\hline Cyanidin-3,5-diglucoside & $29.53 \pm 0.36^{\mathrm{a}}$ & $0.51 \pm 0.09^{\mathrm{d}}$ & $0.16 \pm 0.01^{\mathrm{e}}$ & $2.82 \pm 0.17^{\mathrm{b}}$ & $2.11 \pm 0.10^{c}$ & $0.44 \pm 0.08^{\mathrm{d}}$ \\
\hline Cyanidin-3-glucoside & $268.46 \pm 17.30^{\mathrm{a}}$ & $83.41 \pm 8.49^{c}$ & $42.90 \pm 9.15^{\mathrm{e}}$ & $105.21 \pm 5.19^{b}$ & $107.60 \pm 11.54^{\mathrm{b}}$ & $67.53 \pm 1.81^{\mathrm{d}}$ \\
\hline Cyanidin-3-rutinoside & $44.19 \pm 2.52^{\mathrm{b}}$ & $30.61 \pm 2.49^{\mathrm{d}}$ & $18.61 \pm 4.91^{\mathrm{e}}$ & $30.27 \pm 1.28^{\mathrm{d}}$ & $93.03 \pm 3.20^{\mathrm{a}}$ & $37.31 \pm 5.17^{c}$ \\
\hline Pelargonidin dihexoside & $7.21 \pm 0.45^{\mathrm{a}}$ & $2.89 \pm 0.33^{\mathrm{d}}$ & $1.46 \pm 0.11^{\mathrm{e}}$ & $5.74 \pm 0.52^{\mathrm{b}}$ & $3.85 \pm 0.21^{\mathrm{c}}$ & $2.87 \pm 0.22^{\mathrm{d}}$ \\
\hline Pelargonidin-3-glucoside & $10.76 \pm 0.43^{\mathrm{a}}$ & $3.50 \pm 0.16^{\mathrm{e}}$ & $3.94 \pm 0.15^{\mathrm{d}}$ & $3.63 \pm 0.46^{\mathrm{de}}$ & $6.03 \pm 0.21^{\mathrm{c}}$ & $6.42 \pm 0.11^{\mathrm{b}}$ \\
\hline Peonidin dihexoside & $12.39 \pm 0.90^{\mathrm{bc}}$ & $14.75 \pm 0.81^{\mathrm{a}}$ & $6.79 \pm 0.60^{\mathrm{d}}$ & $11.46 \pm 1.14^{\mathrm{c}}$ & $13.38 \pm 0.38^{\mathrm{b}}$ & $11.79 \pm 0.55^{\mathrm{c}}$ \\
\hline Peonidin-3-glucoside & $40.61 \pm 1.52^{\mathrm{a}}$ & $13.07 \pm 0.74^{\mathrm{c}}$ & $6.58 \pm 0.59^{d}$ & $16.70 \pm 1.79^{\mathrm{b}}$ & $15.72 \pm 0.35^{\mathrm{b}}$ & $12.17 \pm 0.21^{\mathrm{c}}$ \\
\hline Total & $1565.37 \pm 42.52^{\mathrm{a}}$ & $1035.56 \pm 61.54^{\mathrm{c}}$ & $715.94 \pm 57.15^{\mathrm{e}}$ & $1199.52 \pm 74.99^{b}$ & $1141.90 \pm 59.62^{b}$ & $902.06 \pm 49.25^{\mathrm{d}}$ \\
\hline
\end{tabular}


for 66 hours, by only $40 \%$ with berries dried at $65^{\circ} \mathrm{C}$ for 30 hours and by $66 \%$ at $75^{\circ} \mathrm{C}$ for 20 hours (Fig. 2).

\section{Discussion}

Oszmiański et al. (2016) reported that honeysuckle berries mainly consist of water and soluble solids. Selected bioactive compounds start to alter soon after harvest. Particularly, after harvesting, different fruit ingredients are subject to various enzymatic and non-enzymatic reactions. Enzymatic reactions involve some enzymes, which react in the presence of individual compounds and oxygen, resulting in a change in their contents and composition. Other, non-enzymatic processes do not require enzymatic catalysis, but they include three main reaction pathways: Maillard reaction, caramelization and ascorbic acid oxidation (Sanz et al. 2001). The drying process additionally changes the contents of selective primary and secondary metabolites. Water solubility and heat sensitivity are the main two factors that alter the content of selected compounds in dried fruits (Karaman et al. 2014). Honeysuckle berries have extremely firm skins, which impeded water evaporation in our study. We thus needed much more time to dry the berries to an acceptable dryness (85\%) than is needed for other often-dried fruits. FAO (2019) reported the desirable final moisture content in dried fruits to be $15 \%$. In general, heat treatment means water conversion into vapor, which passes in a gaseous state across a disrupted cell and consequently changes the concentration of solid components and the evaporation of some volatile compounds (Karathanos 1999, Yadav and Singh 2014, Karaman et al. 2014). At lower temperatures, the transformation process lasts longer than at higher temperatures, in which water passes from the fruit faster. Enough moisture (85\%) must be removed for the product to be considered dried, otherwise mold starts grow on the berries in a few days (FAO 2019). Singh et al. (2006) found that a low temperature of drying caused minimum damage to dried material, retaining more nutrients in the fruits than other drying methods.

Sugars in blue honeysuckle berries in general decrease with drying. Our sugar contents were in accordance with previous published studies (Oszmiański et al. 2016, Auzanneau et al. 2018). One of the reasons for the decrease in the sugar content was the Maillard reaction. This is a complex series of reactions between amines, amino acids and proteins with sugars and it is the major cause of fruit browning during heating processes. The result of that reaction is reduced sugar content and the formation of brown pigments (Yilmaz and Toledo 2005). The Maillard reaction was not the only reason for sugar reduction. Caramelization also occurs, taking place above the melting point of sugar, which darkens to a brown color and decreases the sugar content (Sanz et al. 2001). Furthermore, heating of the berries caused water evaporation and partial decomposition of sugars i.e. transformation to volatiles, such as water vapor and carbon dioxide, or other types of carbon-containing volatiles (Karatha- nos 1999). The other elements of sugars were modified into crystallized structures (Senica et al. 2016), which only diffuse from the berry with difficulty. All heat treatment caused some extent of water evaporation and the formation of soluble sugars, but a longer duration at higher temperatures can destroy sugar molecules, which are prone to chemical transformation at elevated temperatures (Karaman et al. 2014).

High ascorbic acid content in fresh blue honeysuckle berries is also found in the study of Jurikova et al. (2009). Ascorbic acid content can be affected by many factors like heat intensity, drying time, final moisture content and air velocity (Santos and Silva 2008). Ascorbic acid is a water-soluble compound (Khattab et al. 2017) and it is lost from the berries with vapor. Khattab et al. (2017) reported the reduction of vitamin $\mathrm{C}$ by $90 \%$ at a temperature of $60^{\circ} \mathrm{C}$ following $24 \mathrm{~h}$ drying. Our results are in agreement with Khattab et al. (2017) as ascorbic acid was lost from berries after less than 24 hours of drying at $75^{\circ} \mathrm{C}$. Degradation of ascorbic acid in dried fruit starts with the first-order reaction, followed by the effect of the reduction of the moisture content which, as the process of drying proceeds with the temperature effect, becomes predominant (Santos and Silva 2008, Goula and Adamopoulos 2006, Qiu et al. 2018; Di Scala and Crapiste 2008). The first reactions may depend on water activity, $\mathrm{pH}$ and the presence of degraded enzymes. With an increase of the water content, the aqueous phase becomes less viscous, which enhances diffusion in the media. This facilitates the reaction of oxidation and, consequently, the degradation of certain compounds (Santos and Silva 2008). The same authors also reported that both heat intensity and time of drying have a major effect on ascorbic acid degradation. A longer drying time results in a lower retention of ascorbic acid. In addition, higher temperatures $\left(>65^{\circ} \mathrm{C}\right)$ and the consequent increase in relative humidity resulted in a lower retention of ascorbic acid.

During the drying process, moisture and temperature altered with drying time and their combination caused degradation, and the formation of organic acids. Tartaric, fumaric and shikimic acids increased with both higher temperature and duration of heating. Quinic, citric and malic acids, the most abundant of the organic acids in blue honeysuckle berries seem to be sensitive to drying time (more than 30 hours), but their contents increased with temperatures above $60^{\circ} \mathrm{C}$. It seems that organic acids tolerate higher temperatures better than a long time of heating. Regardless of the temperature, dry matter was similar among treatments. Higher temperatures mean faster water transfer from the berries, which is linked to a higher loss of low-molecular weight components (Kucner et al. 2014, Zorenc et al. 2017), the same as with sugars. On the other hand, organic acid content seems to be more sensitive to a long duration of heating. Lower temperatures $\left(40\right.$ and $50{ }^{\circ} \mathrm{C}$ ) imply low porosity of the epidermal layer of various berries, which is reflected in slower mass transfer, and the destruction of molecules (Kucner et al. 2014). Chen et al. (2012) reported that organic acids, including citric acid, degraded via the gam- 
ma-aminobutyrate shunt pathway following a longer time of heating at lower temperatures.

Blue honeysuckle berries are rich in phenolic compounds and their presence as established here is in accordance with some other studies (Senica et al. 2018a, b; Oszmiański et al. 2016). The last mentioned study put special emphasis on iridoids with high health properties. In our study, significant changes occurred in their contents during the thermal treatment at different heating times and heat intensities. Total phenolic content has been reported to diminish as a result of food processing (Može Bornšek et al. 2015). The main reason for phenolic content decrease is the transfer of phenolics to the hypertonic solution. Heat treatment causes cellular disruption and the exposure of phenolics to oxidative and hydrolytic enzymes (Wojdyło et al. 2009, Karaman et al. 2014). The most important enzymes are polyphenol oxidases (PPO), which catalyze the oxidation of colorless phenolic compounds into o-quinones, which are red to brown in color. Heating and poor handling of fruits or vegetables cause greater PPO activation and, accordingly, a decrease in the content of some phenolic compounds (Sanz et al. 2001, Yilmaz and Toledo 2005). Additionally, with the drying process, a longer time or a higher heat intensity increases the presence of PPO (Kucner et al. 2014). Additionally, Wojdyło et al. (2009) reported that drying temperatures between 55 and $85{ }^{\circ} \mathrm{C}$ reduced the content of phenolics. They suggested that an irreversible oxidative process and prolonged exposure to thermal degradation may be the cause of altered levels of phenolic compounds.

Unlike other groups of phenolics, the content of hydroxycinnamic acids (HCA) increased with both heating time and temperature. That is in agreement with several reports (Brownmiller et al. 2008, Wojdyło et al. 2009, Oszmiański et al. 2016). Zorić et al. (2014) also noted the high heat stability of HCA. Kaneko et al. (2016) reported that selected HCA have extremely high thermomechanical performance. The thermal degradation temperature for HCA was around $300{ }^{\circ} \mathrm{C}$ (Kaneko et al. 2016), which was four times higher than our highest studied drying temperature.

Flavanols, flavonols, flavanones, flavones and isoflavones made up the group of flavonoids. Their heat sensitivity caused by drying depends on the structural stability of the selected compound. In particular, compounds with a double bond in the structure need more energy in order to be degraded (Chaaban et al. 2017). Wang et al. (2000) reported that compounds from the flavanol group, especially epicatechin, are highly sensitive to oxidation processes. In our study, their contents greatly decreased regardless of the temperature and time of heating. Flavonols have two more double bonds in their basic molecular structures than the previously described flavanols. They are therefore more stable during heat treatment. The structure of flavonones resulted in greater stability with respect to the intensity but not with respect to the duration of drying. Degradation of these contents occurred, but more slowly than with some more thermolabile compounds, such as anthocyanins and flavanols (Zorić et al. 2014).
Anthocyanins, with high beneficial properties for human health, are mostly concentrated in the skin of the blue honeysuckle berry (Oszmiański et al. 2016). Accordingly they are more exposed to degradation, including from heating. Total anthocyanins showed significant differences among the various heat treatments (Figure 2). Berries dried at $65^{\circ} \mathrm{C}$ for $30 \mathrm{~h}$ (241.72 $\left.\mathrm{mg} 100 \mathrm{~g}^{-1}\right)$ had the highest anthocyanin content, but still only approximately half that of fresh berries (413.15 mg $100 \mathrm{~g}^{-1}$ ). Berries dried at $50{ }^{\circ} \mathrm{C}$ for 200 $\mathrm{h}$ had the lowest anthocyanin content. Khattab et al. (2016) also found a positive correlation between drying temperature and anthocyanin degradation. Those results are also in accordance with Zorić et al. (2014) and Zorenc et al. (2017), who reported that degradation of anthocyanins is significantly greater at higher temperatures. A higher stability of anthocyanins during heating was achieved by using a lower temperature and shorter duration of heating during processing (Wang and Xu 2007, Zorić et al. 2014). Our study showed that dried berries had the same losses at $40{ }^{\circ} \mathrm{C}$ as at $75^{\circ} \mathrm{C}$, which confirms that heat treatment in itself negatively affects the cyanidin content. Additionally, loss of anthocyanins can be attributed to various factors, such as residual enzyme activity or condensation reactions with other phenolics (Brownmiller et al. 2008). Some anthocyanins are more vulnerable than other phenols from that group, because of their different chemical structure (Srivastava et al. 2007), mainly due to different sugar and hydroxyl moieties. Cyanidin glycosides are considered to be less stable in relation to heating, which is in accordance with our study, in which cyanidin-3-glucoside and cyanidin 3.5-diglucoside was 70 to $98 \%$ lower than in fresh berries. The results showed that peonidin and pelargonidin are more stable in relation to heat, which is in agreement with previous studies (Srivastava et al. 2007, Khattab et al. 2016).

Dried blue honeysuckle berries are a durable and convenient product available throughout the year. It is important to recognize that drying has many advantages; apart from cost reduction, it also accelerates water loss and consequently prevents the growth of bacteria, fungi, and other microorganisms. The chief disadvantage is the loss of some important nutritional compounds. Sagar and Kumar (2010) reported that an optimal drying system for the preservation of fruits should be cost effective, with a short drying time and minimum damage to the food product. The studied contents of some primary and secondary metabolites in our study responded differently to drying conditions. Sugars, flavonols and HCA appeared to be more thermostable substances, especially HCA, which increased by more than $75 \%$ with drying, regardless of the drying time and temperature. On the other hand, anthocyanins and flavanols were highly thermolabile substances, their contents decreasing with an increase of both drying time and temperature. Additionally, ascorbic acid totally degraded with heat treatment. What is more, organic acids seem to be more sensitive to long exposure to drying, than to higher temperature of heating, while iridoids are more sensitive to higher heating temperatures. 
In conclusion, we found that the optimal treatment was drying at $60^{\circ} \mathrm{C}$ for $33 \mathrm{~h}$, which is in agreement with Garba and Kaur (2014). Understanding the structural stability of selected active compounds in blue honeysuckle berries will help their processors to provide high quality dried berry products with rich nutritional properties.

\section{References}

Auzanneau, N., Weber, P., Kosińska-Cagnazzo, A., Andlauer, W., 2018: Bioactive compounds and antioxidant capacity of Lonicera caerulea berries: Comparison of seven cultivars over three harvesting years. Journal of Food Composition and Analysis 66, 8189.

Becker, R., Pączkowski, C., Szakiel, A., 2017: Triterpenoid profile of fruit and leaf cuticular waxes of edible honeysuckle Lonicera caerulea var. kamtschatica. Acta Societatis Botanicorum Poloniae 86, 3539-3548.

Brownmiller, C., Howard, L.R., Prior, R.L., 2008: Processing and storage effects on monomeric anthocyanins, percent polymeric color, and antioxidant capacity of processed blueberry products. Journal of Food Science 73, H72-H79.

Chaaban, H., Ioannou, I., Chebil, L., Slimane, M., Gérardin, C., Paris, C., Charbonnel C., Chekir, L., Ghoul, M., 2017: Effect of heat processing on thermal stability and antioxidant activity of six flavonoids. Journal of Food Processing and Preservation 41, e13203.

Chauhan, A.K.S., Srivastava, A.K., 2009: Optimazing drying conditions for vacuum-assisted microwave drying of green peas (Pisum sativum L.). Drying Technology 27, 761-769.

Chen, M., Jiang, Q., Yin, X.-R., Lin, Q., Chen, J.-Y., Allan, A.C., $\mathrm{Xu}$, C.-J., Chen, K.S., 2012: Effect of hot air treatment on organic acid- and sugar- metabolism in Ponkan (Citrus retuculata) fruit. Scientia Horticulturae 147, 118-125.

Cuce, M., Sokmen, A., 2017: In vitro production protocol of Vaccinium uliginosum L. (bog bilberry) growing in the Turkish flora. Turkish Journal of Agriculture and Forestry 41, 294304.

Çavuşoğlu Ş., 2018: Effects of hot water and UV-C on mineral content changes in two strawberry cultivars stored at different temperatures. Turkish Journal of Agriculture and Forestry $42,423-432$.

Di Scala, K.C., Crapiste, G.H., 2008: Drying kinetics and quality changes during drying of red pepper. LWT-Food Science and Technology 41, 789-795.

Ercisli, S., Tosun, M., Karlidag, H., Dzubur, A., Hadziabulic, S., Aliman, Y., 2012: Color and antioxidant characteristics of some fresh fig (Ficus carica L.) genotypes from Northeastern Turkey. Plant Foods for Human Nutrition 67, 271-276.

FAO, 2019. Fruit processing tool kit; Dried fruit. Retrieved July 10, 2019 from http://www.fao.org/3/a-au111e.pdf.

Garba, U., Kaur, S., 2014: Effect of drying and pretreatment on anthocyanins, flavonoids and ascorbic acid content of black carrot (Daucus carrota L.). Journal of Global Biosciences 3, 772-777.

Garden-Robinson, J., 2012: Drying Fruits. In: Food Preservation. North Dakota State University Extension Service. Retrieved June 4, 2019 from https://www.ag.ndsu.edu/publications/ food-nutrition/food-preservation-drying-fruits.

Goula, A.M., Adamopoulos, K.G., 2006: Retention of ascorbic acid during drying of tomato halves and tomato pulp. Drying Technology 24, 57-64.

\section{Acknowledgement}

The research is part of the program Horticulture No. P4-0013-0481, which is funded by the Slovenian Research Agency (ARRS). The authors would like to thank Haskap d.o.o. for contributting the plant material.

Gündüz K., Özbay, H., 2018: The effects of genotype and altitude of the growing location on physical, chemical, and phytochemical properties of strawberry. Turkish Journal of Agriculture and Forestry 42, 145-153.

Hummer, K.E., Pomper, K.W., Postman, J., Graham, C.J., Stover, E., Mercure, E.W., Aradhya, M., Cristoso, C., Ferguson, L., Thompson, M., Byers, P., Zee, F., 2012: Emerging fruit crops. In: Badnes, M.L., Byrne, D.H. (eds.), Fruit breeding, 97-147. Springer, New York.

Jurikova, T., Matuskovic, J., Gazdik, Z., 2009: Effect of irrigation on intensity of respiration and study of sugar and organic acid content in different development stages of Lonicera ka$m$ tschatica and Lonicera edulis berries. Horticulturae Science $36,14-20$.

Jurikova, T., Sochor, J., Rop, O., Mlček, J., Balla, Š., Szekers, L., Žitný, R., Zitika, O., Adam, V., Kizek, R., 2012: Evaluation of polyphenolic profile and nutritional value of non-traditional fruit species in the Czech Republic - A comparative study. Molecules 17, 8968-8981.

Kaneko, T., Tateyam, S., Okajima, M., Hojoon, S., Takaya, N., 2016: Ultrahigh heat-resistant, transparent bioplastics from exotic amino acid. Materials Today: Proceedings, 3S, S21-S29.

Karaman, S., Toker, O.S., Cam, M., Hayta, M., Dođan, M., Kayacier, A., 2014: Bioactive and physiochemical properties of persimmon as affected by drying methods. Drying Technology 32, 258-267.

Karathanos, V.T., 1999: Determination of water content of dried fruits by drying kinetics. Journal of Food Engineering 39, 337-344.

Khattab, R., Ghanem, A., Brooks, M.S-L., 2016: Stability of haskap berry (Lonicera caerulea L.) anthocyanins at different storage and processing conditions. Journal of Food Research 5, 67-79.

Khattab, R., Ghanem, A., Brooks M. S.-L., 2017: Quality of dried haskap berries (Lonicera caerulea L.) as affected by prior juice extraction osmotic treatment, and drying conditions. Drying Technology 35, 375-391.

Kucner, A., Klewicki, R., Sojka, M., Klewicka, E., 2014: Osmotic concentration of gooseberry fruits - the influence of temperature, time and pretreatment methods on mass transfer and total polyphenol and organic acid content. Food Technology and Biotechnology 52, 411-419.

Mikulic-Petkovsek, M., Slatnar, A., Stampar, F., Veberic, R., 2012: HPLC-MS ${ }^{\mathrm{n}}$ identification and quantification of flavonol glycosides in 28 wild and cultivated berry species. Food Chemistry 135, 2138-2146.

Mikulic-Petkovsek, M., Koron, D.,Veberic, R., 2016: Quality parameters of currant berries from three different cluster positions. Scientia Horticulturae 210, 188-196.

Miyashita, T., Ohashi, T., Shibata, F., Araki, H., Hoshino, Y., 2009: Plant regeneration with maintenance of the endosperm ploi- 
dy level by endosperm culture in Lonicera caerulea var. emphyllocalyx. Plant Cell, Tissue and Organ Culture 98, 291-301.

Može Bornšek, S.M., Polak, T., Skrt, M., Demšar, L., Ulrih N.P., Abram, V., 2015: Effects of industrial and home-made spread processing on bilberry phenolics. Food Chemistry 173, 61-69.

Mundada, M., Hathan, B.S., Maske, S., 2010: Convective dehydration kinetics of osmotically pretreated pomegranate arils. Biosystems Engineering 107, 307-316.

Oszmiański, J., Kucharska, A.Z., 2018: Effect of pre-treatment of blue honeysuckle berries on bioactive iridoid content. Food Chemistry 240, 1087-1091.

Oszmiański, J., Wojdył, A., Lachowicz, S., 2016: Effect of dried powder preparation on polyphenolic and antioxidant activity of blue honeysuckle berries (Lonicera caerulea L. var. kamtschatica). LWT - Food Science and Technology 67, 214-222.

Palíková, I., Valentova, K., Oborna, I., Ulrichova, J., 2009: Protectively of blue honeysuckle extract against oxidative human endothelial cells and rat hepatocyte damage. Journal of Agricultural and Food Chemistry 57, 6584-6589.

Qiu, J., Vuist J.-E., Boom, R.M., Schutyse, M.A.I., 2018: Formation and degradation kinetics of organic acids during heating and drying of concentrated tomato juice. LWT- Food Science and Technology 87, 112-121.

Sablani, S.S., Andrews, P.K., Davies, N.M., Walters, T., Saez, H., Sylamadevi, R.M., Mohekar PR., 2010: Effect of thermal treatments oh phytochemicals in conventionally and organically grown berries. Journal of the Science of Food and Agriculture 90, 769-778.

Sagar V.R., Kumar, S.P., 2010. Recent advances in drying and dehydration of fruits and vegetables: a review. Journal of Food Science and Technology 47, 15-26.

Santos, P.H.S., Silva, M.A., 2008: Retention of vitamin C in drying processes of fruits and vegetables-A review. Drying Technology 26, 1421-1437.

Sanz, M.L., del Castilo, M.D., Corzo, N., Olano, A., 2001: Formation of Amadori compounds in dehydrated fruits. Journal of Agricultural and Food Chemistry 49, 5228-5231.

Senica, M., Veberic, R., Grabnar, J.J., Stampar, F., Jakopic, J., 2016: Selected chemical compounds in firm and mellow persimmon fruit before and after the drying process. Journal of the Science of Food and Agriculture 96, 3140-3147.

Senica, M., Stampar, F., Mikulic Petkovsek, M. 2018a: Blue honeysuckle (Lonicera cearulea L. subsp. edulis) berry; A rich source of some nutrients and their differences among four different cultivars. Scientia Horticulturae 238, 215-221.

Senica, M., Bavec M., Stampar, F., Mikulic-Petkovsek, M., 2018b: Blue honeysuckle (Lonicera caerulea subsp. edulis (Turcz. ex Herder) Hultén.) berries and changes in their ingredients across different locations. Journal of the Science of Food and Agriculture 98, 3333-3342.
Singh, U., Sagar, V.R. Behera, T.K., Kumar, P.S., 2006: Effect of drying conditions on the quality of dehydrated selected vegetables. Journal of the Science of Food and Agriculture 43, 579-582.

Srivastava, A., Akoh, C.C., Yi, W., Fisher, J., Krewer, G., 2007: Effect of storage conditions on the biological activity of phenolic compounds of blueberry extract packed in glass bottles. Journal of Agricultural and Food Chemistry 55, 2705-2713.

Thompson, M.M., 2008: Caprifoliaceae; Lonicera cearulea blue honeysuckle. In: Janik J., Paulini, R.E- (eds.), The encyclopedia of fruit and nuts, 232-235. CABI Publishing, Wallingford, England.

Vijayan, K., Chakraborti, S.P., Ercisli, S., Ghosh, P.D., 2008: $\mathrm{NaCl}$ induced morpho-biochemical and anatomical changes in mulberry (Morus spp.). Plant Growth Regulation 56, 61-69.

Wang, L.-F., Kim, D.-M., Lee, C.Y., 2000: Effects of heat processing and storage on flavanols and sensory qualities of green tea beverage. Journal of Agricultural and Food Chemistry 48, 4227-4232.

Wang, S.Y., Zheng, W., Galletta, J.G., 2002: Cultural system affects fruit quality and antioxidant capacity in strawberries. Journal of Agricultural and Food Chemistry 50, 6534-6542.

Wang, W.-D., Xu S.-Y., 2007: Degradation kinetics of anthocyanins in blackberry juice and concentrate. Journal of Food Engineering 82, 271-275.

Wojdyło, A., Figiel, A., Oszmiański, J., 2009: Effect of drying methods with the application of vaccum microwaves on the bioactive compound, color, and antioxidant activity of strawberry fruits. Journal of Agricultural and Food Chemistry 57, 1337-1343.

WFO, 2019: Lonicera caerulea var. edulis Turcz. ex Herder. Published on the Internet; Retrieved December 18, 2019 from http://www.worldfloraonline.org/taxon/wfo-0001224270.

Yadav, A.K., Singh, S.V., 2014: Osmotic dehydration of fruits and vegetables: a review. Journal of Food Science and Technology $51,1654-1673$.

Yilmaz, Y., Toledo, R., 2005: Antioxidant activity of water-soluble Maillard reaction products. Food Chemistry 93, 273-278.

Zorenc, Z., Veberic, R., Stampar, F., Koron, D., Mikulic-Petkovsek, M., 2017: Thermal stability of primary and secondary metabolites in highbush blueberry (Vaccinium corymbosum L.) purees. LWT-Food Science and Technology 76, 79-86.

Zorić, Z., Dragović-Uzelac, V., Pedisić, S., Kurtanjek, Ž., Garofolić, I.E., 2014: Kinetics of the degradation of anthocyanins, phenolic acids and flavonols during heat treatments of freezedried sour cherry marasca paste. Food Technology and Biotechnology 52, 101-108. 